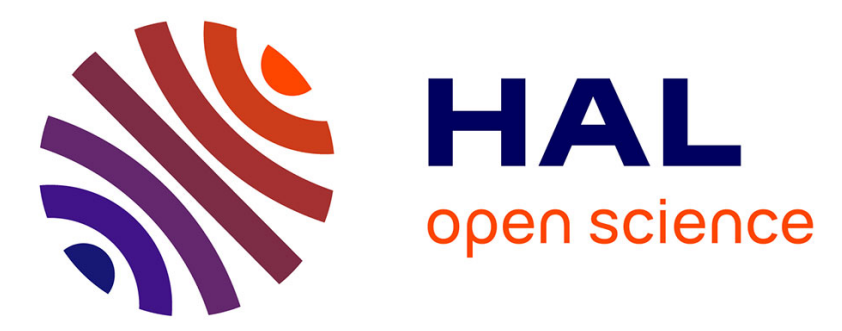

\title{
Sequential updating of a new dynamic pharmacokinetic model for caffeine in premature neonates
}

\author{
Sandrine Micallef, Billy Amzal, Véronique Bach, Karen Chardon, Pierre
}

Tourneux, Frédéric Y. Bois

\section{- To cite this version:}

Sandrine Micallef, Billy Amzal, Véronique Bach, Karen Chardon, Pierre Tourneux, et al.. Sequential updating of a new dynamic pharmacokinetic model for caffeine in premature neonates. Clinical Pharmacokinetics, 2007, 46 (1), pp.59-74. 10.2165/00003088-200746010-00003 . ineris-00961910

\section{HAL Id: ineris-00961910 \\ https://hal-ineris.archives-ouvertes.fr/ineris-00961910}

Submitted on 20 Mar 2014

HAL is a multi-disciplinary open access archive for the deposit and dissemination of scientific research documents, whether they are published or not. The documents may come from teaching and research institutions in France or abroad, or from public or private research centers.
L'archive ouverte pluridisciplinaire HAL, est destinée au dépôt et à la diffusion de documents scientifiques de niveau recherche, publiés ou non, émanant des établissements d'enseignement et de recherche français ou étrangers, des laboratoires publics ou privés. 


\section{Sequential updating of a new dynamic pharmacokinetic model for caffeine in premature neonates}

Short title: Updating of a pharmacokinetic model for caffeine

Sandrine Micallef', PhD., Billy Amzal', PhD, Véronique Bach', PhD, Karen Chardon ${ }^{3}$, PhD, Pierre Tourneux ${ }^{4}, \mathrm{MD}$, and Frédéric Y. Bois ${ }^{1}, \mathrm{PhD}$.

${ }^{1}$ Institut National de l'Environnement Industriel et des Risques, Verneuil-en-Halatte, France.

${ }^{2}$ NOVARTIS PHARMA AG. Basel, Switzerland.

${ }^{3}$ Université Picardie Jules Verne Amiens, France

${ }^{4}$ Neonatal Unit and Intensive Care of the Pediatric Department of the Hospital of Amiens, France.

\section{Acknowledgments}

The authors wish to thank Dr. C. Diack for his helpful remarks and the anonymous referees for comments that improved our manuscript. Partial funding for this work was provided by the French Ministry of Research and Technology, project DIADEME, decision 02 C 0141. This work has also been supported by the research project BCRD-AP2001-DRC07 provided by the French Ministry of the Ecology and Sustainable Development and by the Regional Council of Picardie.

Corresponding author: Sandrine Micallef,

Experimental Toxicology Unit,

EA 3901 TOXI-DMAG,

Institut National de l'Environnement Industriel et des Risques,

Parc Technologique ALATA, BP 2,

60550 Verneuil en Halatte, France.

Tel: + $33(0) 344556168$;

Fax: + $33(0) 344556605$;

E-Mail: sandrine.micallef@ineris.fr

Category: Original Research Article.

Word count : 6391 


\section{Table of contents}

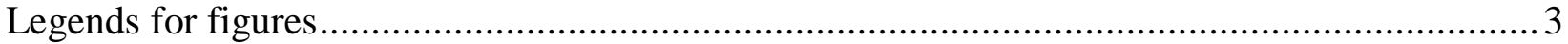

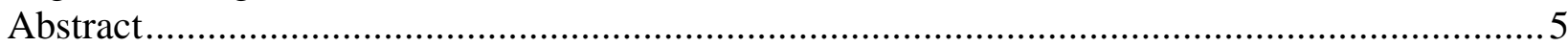

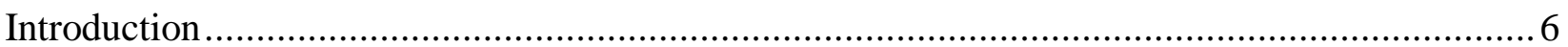

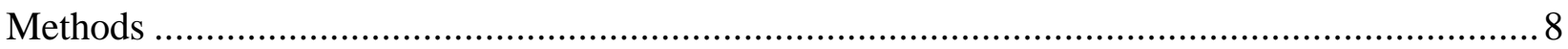

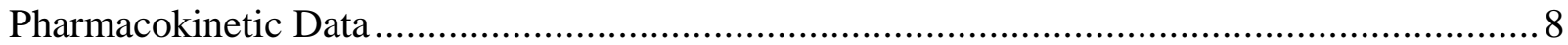

A Dynamic Model of Caffeine Biodistribution in PrematureNeonates .................................9

One-Compartment Caffeine PK Model ............................................................. 9

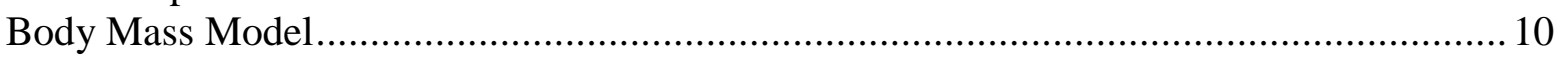

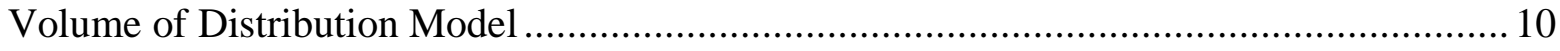

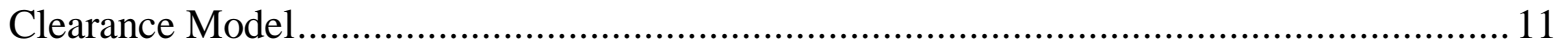

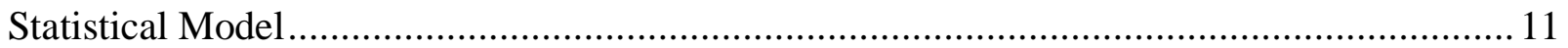

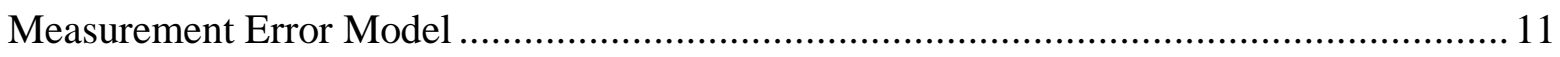

Population Model ......................................................................................... 12

Bayesian Inference via MCMC ......................................................................... 13

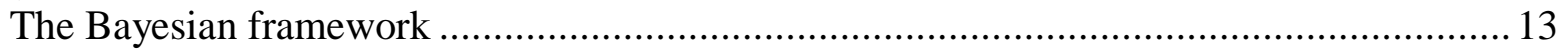

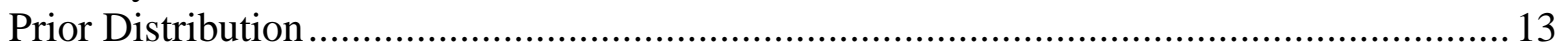

Use of data for model calibration and adequacy checking ........................................... 15

Model Calibration via MCMC ......................................................................... 15

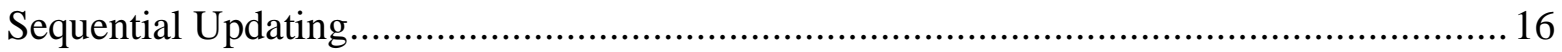

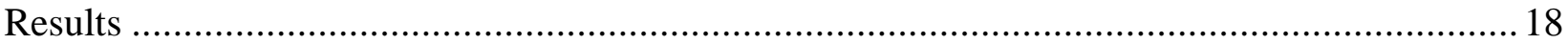

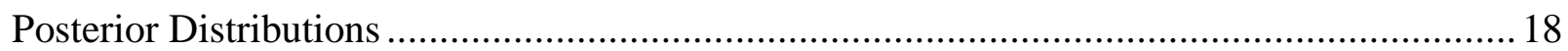

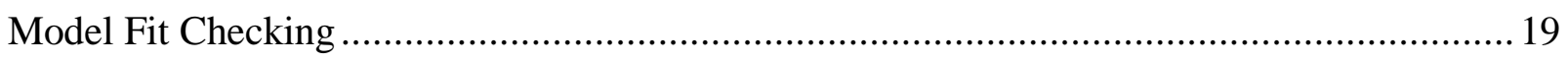

Model Adequacy assessment .............................................................................. 19

Comparison of Predictions from Previously Published Caffeine PK Models ....................... 20

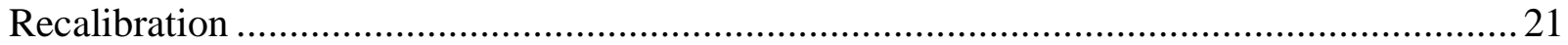

Sequential Updating of Predictions Using Sequential Incoming Data...............................2 21

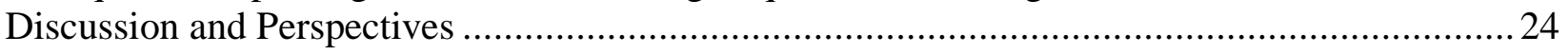

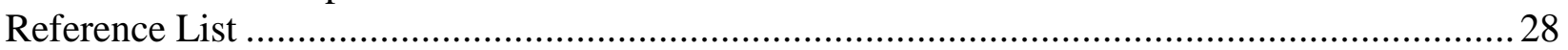

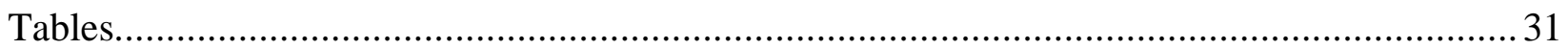




\section{Legends for figures}

Figure 1: Observed caffeine concentrations in blood as a function of postnatal age. Joined points indicate observations belonging to a same individual .

Figure 2: Directed acyclic graph of the hierarchical statistical model. Symbols are: $P$, prior distributions; $\mu_{\theta}$, population mean parameters; $\sigma_{\theta}$, variance of population parameter; $t$, observation times; $\theta$, unknown parameters; $A_{0}$ and $M_{0}$ are respectively duration of gestation and body mass at birth (both supposed exactly measured); $X$, the administered treatment; $M_{t}$, the measured body mass at time $t ; c v_{M}$, the variation coefficient of masses and $Y_{t}$, the measured caffeine concentration in blood. $\mu_{c v M}$, and $\sigma_{c v M}$ are respectively for mean and variance of $c v_{M}$ parameters and $\sigma_{c}$ for the variance of the experimental measurements. $F$ is the dynamic biodistribution model. Square nodes are for variables of known (or supposed known) values; circular nodes for unknown variables and the triangle represents a deterministic link.

Figure 3: Data versus model predictions. The left and the right graphs respectively concern body masses and caffeine concentrations in blood for individuals of the training set. On the right side graph, each line segment describes the $90 \%$-credibility interval associated to the corresponding prediction. The 90\%-crediblity intervals have been plotted using $\sigma_{c}$ value of the maximum posterior vector.

Figure 4: Caffeine concentration in blood (left) and body masses (right) as a function of time. Predictions have been calculated for one subject of the training set (Subject 9) and one subject of the test set (Subject 2). Means of the predictions are in bold lines, and the corresponding 90\%credibility intervals are plotted with thin lines. Data are represented by dots. Note that $90 \%$ credibility interval of the predicted masses is not represented for the subject of the training set (subject 9) as it is very thin. 
Figure 5 : Relative residuals for caffeine concentration as a function of postnatal age. Four different models are represented: Thomson et al. ${ }^{[1]}$ (triangles), Lee et al. ${ }^{[2]}$ (squares), Falcao et al. ${ }^{[3]}$ (empty circles) and the one we propose (plain circles). Relative residuals correspond to the relative differences between test set data points and mean caffeine concentration predictions obtained by Monte Carlo simulations.

Figure 6: Body mass predictions as a function of the time for patient 31 . The $5 \%$, and $95 \%$ quantiles of predictions are plotted using gray lines, the mean of predictions using dark bold lines. Data are represented by the dots. Panel A shows the predicted masses calculated from the original posterior-sampled parameters, using the covariates of the patient at birth, only. Predictions of panel B use, in addition, body mass data via the updated posterior parameters obtained by a particle algorithm.

Figure 7: Caffeine concentration predictions as a function of time for patient 31 . The $5 \%$ and 95\% quantiles of predictions are plotted using thin lines, the mean of predictions using bold lines. The data point is represented by dot. Panel A shows the predicted caffeine concentration in blood calculated from original posterior sampled parameters and using only the covariates of the patient. Predictions of panel $\mathrm{B}$ and $\mathrm{C}$ are performed using the posterior parameters updated via a particle algorithm. In panel B, only data masses are used for parameter updating while only measured caffeine concentration is used to update predictions in panel $\mathrm{C}$.

Figure 8: Histograms of the marginal posterior distributions of the population elimination model parameters. Top row graphs give the marginal distributions of the original posterior. Bottom row darker histograms give the marginal updated distributions, using one caffeine concentration data point for subject 31. Bottom row unfilled histograms recall the original posterior. 


\section{Abstract}

Background: Caffeine treatment is widely used in nursing care to reduce the risk of apnea of premature neonates. To check the therapeutic efficiency of the treatment against apnea, caffeine concentration in blood is an important indicator. The present study aims at building a pharmacokinetic model as a basis for a medical decision support tool. Methods: A new dynamic pharmacokinetic model is proposed to predict caffeine disposition in premature neonates. Time dependence of physiological parameters is introduced to describe rapid growth of neonates. To take into account the large variability in the population, the pharmacokinetic model is embedded in a population structure. The whole model is inferred within a Bayesian framework. To update caffeine concentration predictions as data of an incoming patient are collected, we propose a fast method such it can be used in a medical context. This involves the sequential updating of model parameters (at individual and population levels) via a stochastic particle algorithm. Results: Our model provides better predictions than the ones obtained with models previously published. We show through an example that sequential updating improves predictions of caffeine concentration in blood (reduce bias and length of credibility intervals.) The update of the pharmacokinetic model using mass and caffeine concentration data is studied. It shows how informative caffeine concentration data are in contrast to mass data. Conclusion: This study provides the methodological basis to anticipate caffeine concentration in blood, after a given treatment if data are collected on the treated neonate or not.

Keywords: Pharmacokinetic compartmental model, population model, Bayesian inference, MCMC algorithms, particle algorithms, caffeine, neonates 


\section{Introduction}

Premature neonates suffer from various physiological deficiencies due to their immaturity at birth. In particular, they can suddenly stop breathing (apnea) spontaneously and quite frequently ${ }^{[4]}$. To reduce the occurrence of apneas, a caffeine treatment is often administered to premature neonates ${ }^{[5-7]}$. This treatment may last several weeks and can induce various secondary effects (on ventilation, sleep, metabolism, weight, glomerular filtration, etc. ${ }^{[8-12]}$. Unfortunately, pediatricians have little knowledge about the actual occurrence of these effects, which appears to be widely variable from an individual to another. To balance treatment benefits and adverse effects, pediatricians try to keep caffeine blood concentration within a "therapeutic" interval. This is checked via blood sampling. Since sampling blood causes trauma on such fragile infants, the number of blood samples is limited. Therefore, it is not possible to collect extensive information on caffeine concentration time course in a given patient and pediatricians refer to a standard protocol of administration ${ }^{[13,14]}$. In doing so, they control treatments or times to sample "on average" in the patients' population. The aim of this paper is to define a PK model to be used as a basis for a medical decision tool to help pediatricians in individualizing caffeine administration. The developed PK model allows the predictions of caffeine time-course in a new patient for whom only easily collected covariates (duration of gestation and body mass at birth), and possibly, a few caffeine concentration data would be available. For this, we need to take advantage of all available information: data extracted from medical files, pediatricians' experience and published data on neonate physiology and caffeine population pharmacokinetics ${ }^{[1-}$

3]. In this context, a Bayesian approach is efficient, as it allows an easy aggregation of such widely different and uncertain information sources ${ }^{[15-17]}$ and naturally allows sequential learning. 
To describe caffeine concentration in blood, a compartmental PK model which is an extention of the one proposed by Amzal et al. ${ }^{[18]}$, is proposed. As caffeine is hydrophilic and distributes in body water ${ }^{[19]}$, our pharmacokinetic model has only one compartment. Its main parameters are the volume of distribution and the caffeine clearance. Moreover, as volume of distribution (depending on the amount of total body water) and metabolism change significantly during the few weeks of the treatment ${ }^{[20-22]}$, we propose a dynamic model with time-dependent parameters. To take into account inter-individual variability, a hierarchical population model is defined and calibrated using historical data.

As caffeine elimination is widely variable in infant populations ${ }^{[1-3]}$, a more efficient PK model need to integrate new information on the treated patient. We show here how caffeine concentration predictions of the treated patient can be updated by running a particle algorithm using recorded body mass and/or caffeine concentration data. The proposed algorithm has the advantage to update both individual and population parameters on the basis of new individual data. Therefore there is no need to re-perform time-consuming calibration process to estimate new population parameters when incorporating this new information.

To our knowledge, any tool for caffeine dose individualization is not used in pediatric services, until now. Even if methods for dose individualization have been proposed in the literature for other drugg ${ }^{[23-25]}$ none has been applied to caffeine dosing. We show here how a population pharmacokinetic model can be calibrated using a particle algorithm in order to guide individual caffeine dosing in a reasonable time. Formal optimization of the treatment is not treated; we refer to Amzal et al. ${ }^{[18]}$ and references therein for details on that question.

In the present study, we start by the modeling of the caffeine bio-distribution, explaining how physiological parameters evolve with time. The following section is devoted to defining the 
statistical model, to quantify sources of uncertainty. It also describes the Bayesian framework in which the inference problem is casted, recalling its principle and defining the chosen prior distributions. The next section details the model checking and model adequacy procedures used. We demonstrate, in the last section, how the model predictions can be sequentially updated with incoming data on new treated patients, and how this can be used for designing a medical decision support software. Finally, this approach and its results are discussed and put in perspective.

\section{Methods}

\section{Pharmacokinetic Data}

The pharmacokinetic data come from medical files of 35 neonates treated in the Neonatal Unit and Intensive Care of the Pediatric Department of the Hospital of Amiens (France). These files are the only available in the set of medical files of the neonates admitted in the nursing care department during years 1998, 1999 and 2003. Files were pre-selected such they meet the following requirements:

- contain at least one caffeine concentration measurement,

- $\quad$ the treatment lasts 3 days at least,

- $\quad$ the treatment goes at least until the post conceptional age of the neonates is 35 weeks.

The typical treatment administered to neonates consists of a charging injected dose of $20 \mathrm{mg} / \mathrm{kg}$ possibly spread out on the first two days, then a regular oral dose of $5 \mathrm{~m} / \mathrm{kg}$ every day from the third day. The treatment usually lasts until the post conceptional age of the neonates reaches 35 weeks. Naturally, these doses could have been modified by the pediatricians for clinical reasons. Observed concentrations of caffeine in blood were collected as requested by the physician. It does not exist any sampling scheme (see Figure 1). 
[Figure 1 about here]

For each patient, the extracted data $Y_{t}$ consisted of one to four concentrations of caffeine measured in blood after specific treatment $X$ (timing, injected concentration or quantity given per $o s$, ) and measured daily body masses, $M_{t}$. An example of collected data (for two patients) is given in Figure 4; the observed caffeine concentrations in blood for all patients are represented on Figure 1. Covariates such as duration of gestation $A_{0}$ and body mass at birth $M_{0}$ were also recorded. These data are available upon request.

The average of the body masses at birth for our neonates population $(N=35)$ is $1.34 \mathrm{~kg}$ with a standard deviation of $0.35 \mathrm{~kg}$. The smallest recorded masse at birth is $0.660 \mathrm{~kg}$ while the largest is $2.170 \mathrm{~kg}$. The duration of gestation of these premature neonates is 29.1 weeks long on average (with a standard deviation of 2.1 weeks and a range of $[23,32]$ weeks of gestation).

\section{A Dynamic Model of Caffeine Biodistribution in PrematureNeonates}

\section{One-Compartment Caffeine PK Model}

According to published pharmacokinetic studies ${ }^{[1-3]}$, caffeine distribution in the body is well described by a one-compartment model ${ }^{[19]}$. The body is therefore modeled by one homogeneous compartment of volume $V(t)$ at time $t$. Note that the time origin $(t=0)$ corresponds here to birth time. Caffeine is eliminated from the central compartment with clearance $C L(t)$. The time-course of caffeine concentration $c(t)$ (in $\mathrm{mg} / \mathrm{L}$ ) is therefore described using a first-order elimination. Input drug is modeled as an IV bolus using a zero-order absorption even in case of oral dosing (the assumption here is that absorption is very rapid). This assumption is made because necessary data to identify absorption rate are incomplete. 


\section{Body Mass Model}

From simple examination of the body mass data, it appears that the growth of premature neonates can be, in a first approximation, divided into two distinct periods. The body mass decreases during the first period of length $\tau$, while it subsequently increases during the second period. We propose linear relationships to describe that variation during the two periods. These functions depend on the covariates $M_{0}$ (body mass at birth), $A_{0}$ (post-conceptional age at birth supposed exactly known) and are specific to each individual:

$$
\begin{aligned}
& M(t)=M_{0}+\alpha_{1} t \quad \text { if } \mathrm{t} \leq \tau \\
& M(t)=M_{0}+\alpha_{2} \varangle-\tau \text { - if } \mathrm{t}>\tau
\end{aligned}
$$

where $\alpha_{1}$ and $\alpha_{2}$ are the slopes of the straight line respectively decreasing and increasing of each phases. $M_{\tau}$ corresponds to the body mass at $\tau\left(M_{\tau}=M_{0}+\alpha_{1} \tau\right)$.

\section{Volume of Distribution Model}

The volume of distribution is assumed to vary linearly with body mass:

$$
\begin{aligned}
& V(t)=M(t) \boldsymbol{f}_{25}+\beta \mathrm{A}_{0}+t-T_{25} \tau_{+} \quad \text { if } \mathrm{t} \leq \tau \\
& V(t)=M(t) f_{\tau} \text { if } \mathrm{t}>\tau
\end{aligned}
$$

where $f_{25}$ is defined as the ratio of the distribution volume over body mass at post-conceptional age 25 weeks (time $\left.T_{25}\right)$ and $f_{\tau}$ is the value of that ratio at time $\tau\left(f_{\tau}=\frac{V(\tau)}{M_{\tau}}\right) . \beta$ is the slope of the variation of the volume-over-mass ratio as a function of time. It is defined as follows:

$$
\beta=M_{0} \frac{f_{\tau}-f_{25}}{A_{0}+\tau-T_{25}}
$$

The slope $\beta$ is set such as the ratio volume over mass at post-conceptional age 25 weeks is $f_{25}$ and as the ratio volume over mass at $\tau$ is $f_{\tau}$. As the degree of plasma protein binding is quite low for caffeine ${ }^{[26]}$, the 
volume of distribution is assumed comparable to total body water. In this case, $f_{25}$ and $f_{\tau}$ are approximately equal to fraction of water at time $T_{25}$ and $\tau$.

\section{Clearance Model}

Several authors showed an increase in elimination of caffeine with postnatal age ${ }^{[21,27,28]}$. According to all of them, caffeine elimination increases as a function of time and reaches a plateau after 3 to 4.5 months. Taking into account the duration of treatment (less than two months in general), we only modeled the first period and assumed that caffeine clearance increases linearly with time with a slope parameter $\gamma$ and a constant at origin $(t=0) C L_{0}$ :

$$
C L(t)=C L_{0}+\gamma t
$$

\section{Statistical Model}

The statistical model quantifies both uncertainties (on data measurements as well as modeling) and sources of variability. Data measurements as well as modeling and intra-individual variability errors are taken into account by using a measurement error model. To model uncertainty on parameters and their variability, probability distributions are used. Inter-individual variability is

quantified in the framework of a population model ${ }^{[29,30]}$ by estimating its population parameters. Given the above bio-distribution model, the caffeine concentration in blood of a given patient is conditioned by the covariates $M_{0}$ and $A_{0}$ and the parameter vector $\theta=\left(\alpha_{1}, \alpha_{2}, \tau, f_{\tau}, f_{25}, C L_{0}, \gamma\right)$.

\section{Measurement Error Model}

The caffeine concentration in blood actually observed is affected by measurement errors which are assumed to be independent and log-normally distributed with a geometric mean of one and a geometric standard deviation $\sigma_{c}$. Likelihood is then given by: 


$$
\log (Y) \sim N \log (F(X, \theta)), \sigma_{c_{-}}^{-}
$$

where the function $F$ corresponds to the PK model described above.

A normal distribution (with zero mean and standard deviation $\sigma_{M}$ ) measurement error model is also specified for body masses $M_{t}$ :

$$
M(t) \sim N \mathbb{M}(t), \sigma_{M}
$$

To take into account inter-individual variability of body masses (due, for example, to urinary excretion), the standard deviation of measured body masses has been considered as individualspecific. It has been chosen as depending on the body mass of the neonate and on the individual parameter $c v_{M}$ :

$$
\sigma_{M}=c v_{M} \times M_{t}
$$

\section{Population Model}

The pharmacokinetic model has been embedded in a population model which considers that each subject parameter values $\theta$ are drawn from a statistical distribution $G$ with given population mean $\mu_{\theta}$ and variance $\sigma_{\theta}$ (Figure 2).

[Figure 2 about here]

The proposed population model has two major components: the individual and the population levels. At the individual level, parameters $\theta$ are assumed to be normally or log-normally distributed (see Table I) with population means $\mu_{\theta}$ and standard deviations $\sigma_{\theta}$. Truncation bounds of the distributions are set on the basis of the limits for plausible values. For each subject, the expected values of body mass and caffeine concentration in blood are a function $F$ of administration $X$, depending on parameter $\theta$, and the individual's covariates. At the population level, $c v_{M}$ is assumed to be distributed according to a truncated normal distribution with mean $\mu_{c v M}$ and standard deviation $\sigma_{c v M}($ Table I). 
[Table I about here]

\section{Bayesian Inference via MCMC}

\section{The Bayesian framework}

The population model described above is fitted to observed data, $Y_{t}$, with Bayesian techniques ${ }^{[16,}$ 17]. The Bayesian approach yields a sample $\theta$ of parameter values from their joint posterior distribution. From Bayes' theorem, the joint posterior distribution of parameters is proportional to the prior distributions of parameters multiplied by the data likelihood. The posterior is then an update, using collected data, of what it is known about parameters prior to the experiment.

\section{Prior Distribution}

The first step of the Bayesian approach consists in defining prior distributions that quantify the information coming from pediatricians expertise or from the scientific literature. Expertise of clinicians is then entered in the model through its structure and through the use of prior distributions parameters. Prior distributions on population parameters and their associated parameters are summarized in Table I. On the basis of available information, we specify a prior that covers the range of value that is deemed reasonable for each parameter. When we deal with mean parameters, we use truncated normal or uniform distributions as prior while for standard deviation parameters truncated lognormal or loguniform laws are used. For standard deviations, lognormal prior distributions are chosen when little prior knowledge about the parameter is available. For parameters on which no prior information is found, loguniform distribution is used. This distribution is preferred to the more standard Inverse Gamma distribution since it does not require to define mean and variance (as Inverse Gamma does) and only uses truncation bound. 
For parameters related to body mass $\left(\alpha_{1}, \alpha_{2}, \tau\right)$, priors are elicited ${ }^{[31-33]}$ by encoding the pediatricians' knowledge. From their experience, neonates loose, on average about $25 \mathrm{~g} /$ day during between the 3 to 20 first days, then gain bout $25 \mathrm{~g} /$ day. This is used to build priors on $\boldsymbol{\Lambda}_{\alpha_{1}}, \mu_{\alpha_{21}}, \mu_{\tau}$. Information on $\boldsymbol{}_{\alpha_{1}}, \sigma_{\alpha_{21}}, \sigma_{\tau}$, are less easily elicited and rather flat priors are used.

Since the degree of plasma protein binding is quite low for caffeine, we assume that the ratio volume over mass is approximately equal to fraction of body water. Thus, for $f_{25}$ and $f_{\tau}$, priors are proposed after referring to the percentage of water in body reported by the International Commission on Radiological Protection ${ }^{[20]}$. For parameters related to clearance, $C L_{0}$ and $\gamma$, prior distributions are defined after re-parameterization of values from the literature ${ }^{[1-3]}$. Globally, all the previous studies agree and give the same magnitude order for the mean of the clearance at birth, $\mu_{C L_{0}}$ (estimated between 0.006 and $0.03 \mathrm{~L} / \mathrm{h}$ with an estimated standard deviation around 0.003 ) and for the shape of temporal evolution of clearance $\mu_{\gamma}$ (estimated between $1 \mathrm{e}^{-6}$ and $5 \mathrm{e}^{-5}$ with an estimated standard deviation around $3 \mathrm{e}^{-6}$ ). We have chosen mean of $\mu_{C L_{0}}$ and $\mu_{\gamma}$ such they are a compromise between the published studies. To be not too restrictive and to take into account possible differences between studied populations, standard deviations are chosen a bit higher than those estimated in the literature. Population variance of clearance parameters $\left(\sigma_{C L_{0}}{ }^{2}\right.$ and $\sigma_{\gamma}{ }^{2}$ ) are assigned prior distributions truncated between 1.1 and 3 corresponding to a population variability comprised between 10 and 300\%: less than10\% of variability for a clearance parameter in the population seems incredible and 300\% seems large enough since interindividual variability of caffeine clearance was estimated to be around $80 \%$ for adults ${ }^{[34]}$ and is likely a bit larger for neonates. 


\section{Use of data for model calibration and adequacy checking}

The data are used to calibrate (fit), check and assess adequacy of the model. The adopted approach for model adequacy checking is cross-validation ${ }^{[35,36]}$. To that effect, data files are divided into two groups: the training set and the test set.

We start with the training set for model calibration (estimation of the different parameters) and checking the fits. Then, next we use the calibrated model to check its adequacy with respect to the test set. We also perform another evaluation of the model which consists in calculating the percentages of caffeine concentration and body mass data points from the test set falling within different credibility intervals for predictions. The results of these tests are discussed further below.

Both sets of data are formed by a random binomial assignment with parameters equal to $(p=1 / 3$, $N=35$ ). Twenty-four medical files are drawn for the training set (calibration and checking the fits) and the other eleven files are assigned to model adequacy checking (test set).

This process allows us to validate the model. Once this is done, we recalibrate the model using the full data set (35 subjects) to take into account all available information in our prediction procedure. This recalibration step gave what we call in the sequel, original posterior distribution.

\section{Model Calibration via MCMC}

We assessed the model parameters by sampling their values from the joint posterior distributions of all population and individual parameters, using the data from the 24 training set subjects. For this, we used Markov Chains Monte-Carlo simulations ${ }^{[37,38]}$ (Metropolis-Hastings sampler) performed by MCSim software ${ }^{[39]}$ version 5.0.0. After 40,000 iterations of 3 parallel Markov chains, their convergence is checked by calculating the Gelman and Rubin $\hat{R}$ ratio on the 20, 000 last iterations ${ }^{[16]}$. The highest $\hat{R}$ computed is 1.12 , showing that all chains had approximately 
converged. To get a sample from the targeted posterior distribution, we took one in 10 vector out additional 20,000 iterations of each chain after convergence checking (iteration 40,000 to 60,000) leading to a posterior sample of 6,000 parameter vectors. Running the three MCMC chains took about one day (on a pentium 4, microprocessor PC $2.8 \mathrm{GHz}$ ).

\section{Sequential Updating}

For an incoming subject (just after birth), parameters can be guessed on the basis of population distribution (i.e., by sampling from those distributions). However, when data are collected on a subject during treatment, the parameters for that individual can be updated. For parameter updating, it is possible to re-perform the calibration process described above using all available data including the newly recorded ones. This involves re-running Markov chains. But this procedure is not applicable in practice by pediatricians because it is time-consuming. Thus, we propose to apply a particle algorithm ${ }^{[40-43]}$ to sequentially update the posterior (individual and population) parameter sample obtained by calibration (original posterior). Since they start from the original posterior rather than from the priors of Table I, calculations converge faster than the classical calibration procedure $^{[44]}$. Individual parameters and population parameters can be jointly and quickly updated. Other alternative methods to standard MCMC calibration have been proposed (MAP Bayesian, Multiple Model, and Interacting Multiple Model ${ }^{[29,45]}$ to update predictions of the new patient. Our method generalizes MAP approach (consider the whole distribution instead of maximum a posteriori) and allows the updating of population parameters which was not proposed with MM or IMM methods, to our knowledge. The updating of population parameters would allow predictions on another future patient using the information brought by the incoming one. 
The proposed updating method assumes that a current sample is available and consists in few steps. Call $\left(\theta_{l}, \ldots, \theta_{N}\right)$ our original posterior sample of parameters, which approximate a distribution $p(\theta)$, and let's denote $y$ the new coming data. Each $\theta_{i}$ is also called a "particle" in this framework. We want to transform our sample into an another one (of same size $N$ ) from $p(\theta \mid y)$. The detailed updating algorithm can be described as follows:

- For each particle $i=1 . . N$, compute its normalized weight $w_{i}$ (as in the Sampling Importance Resampling scheme of Rubin ${ }^{[46,47]}$

$$
w_{i} \propto \frac{p\left(\theta_{i} \mid y\right)}{p\left(\theta_{i}\right)} \propto p\left(y \mid \theta_{i}\right)
$$

These weights are easy to compute since they take the form of likelihood terms normalized by the sum of all likelihood.

- Resample $N$ particles $\left(\theta^{\prime}{ }_{1}, \ldots, \theta^{\prime}{ }_{N}\right)$ from $\left(\theta_{1}, \ldots, \theta_{N}\right)$ according to the multinomial distribution $M\left(N, w_{1}, \ldots, w_{N}\right)$

- To avoid degeneracy problems, enrich the sample using a Markov kernel $Q$ whose stationary distribution is $p(\theta \mid y)$. For each $i=1 . . N$, we draw $\left(\theta^{\prime \prime}{ }_{1}, \ldots, \quad \theta^{\prime \prime}{ }_{N}\right)$ from $\left(\theta^{\prime}{ }_{1}, \ldots, \theta^{\prime}{ }_{N}\right)$. By definition, this transforms an approximated sample $\left(\theta^{\prime}{ }_{1}, \ldots, \theta^{\prime}{ }_{N}\right)$ from $p(\theta \mid y)$ into another sample $\left(\theta^{\prime \prime}{ }_{1}, \ldots, \theta^{\prime \prime}{ }_{N}\right)$ from $p(\theta \mid y)$, but now richer (more diverse). This final sample is the updated parameter sample.

A convenient choice for the Markov step $Q$ is a Metropolis-Hastings move as used in standard MCMC ${ }^{[37,38]}$. We chose a Metropolis-Hasting step with an independent sampler ${ }^{[48]}$ in which candidates for population parameters are drawn from the original posterior sample and individual parameter from population distribution. To perform updating procedure, we use Matlab software. 


\section{Results}

\section{Posterior Distributions}

The MCMC-calibrated model has been first checked by analyzing the initial posterior vectors' sample (data not shown, see Table II for a summary of final posterior estimate).

Means of mass model parameters $\mu_{\alpha 1}$ and $\mu_{\alpha 2}$ were quite well appraised a priori by the experts, but calibration has decreased the standard deviation of these two parameters. Premature neonates lose, on average, 30 grams per day until the end of the sixth day, then gain, in a second period, 23 grams per day on average. Body mass loss and the length of time during which decrease occurs are quite variable between individual (around $40 \%$ for both). The values of body weight gain are less variable in this population (around 25\%).

Posterior means of the population parameters related to the volume of distribution $\left(\mu_{f 25}\right.$ and $\left.\mu_{f \tau}\right)$ are close to the priors. This is partly due to the very informative nature of the prior distribution for these two parameters. The mean of the volume-over-mass ratio in population is equal to $85 \%$ at post-conceptional age 25 weeks and $75 \%$ at time $\tau$. In parallel, calibration has increased knowledge on population variability (The CVs of $\sigma_{f 25}$ and $\sigma_{f \tau}$ decreased from $20 \%$ to $16.3 \%$ and $15.3 \%$ respectively).

On average, clearance at birth is about $0.3 \mathrm{~L} /$ day. For our neonates (mean body mass: $1.340 \mathrm{~kg}$ and mean distribution volume: $85 \%$ of mass), the half-life of caffeine is $t_{1 / 2}=2.6$ days. Clearance at birth, $C L_{0}$, and slope of the temporal linear evolution of clearance, $\gamma$, are quite variable in this population (CV of $C L_{0}$ about $27 \%$ and $\mathrm{CV}$ of $\gamma$ equal to $77 \%$ ). 


\section{Model Fit Checking}

Figure 3 shows model-predicted values using the joint maximum posterior parameter vector versus their observed counterparts.

[Figure 3 about here]

The posterior sample gives quite a good fit to the body mass observations. The average of the relative errors for mass is about $0.1 \%$ with a minimum around $-12.4 \%$ and a maximum of $14 \%$. Predicted caffeine concentrations in blood are not that good; the relative error is $8.5 \%$ in average with a minimum and a maximum respectively equal to $-108 \%$ and $48 \%$. Figure 4 shows the average of predicted caffeine concentrations and body mass predictions over time, for one subject of the training set (Subject 9). As already observed on Figure 4, model predictions well describe the data.

[Figure 4 about here]

\section{Model Adequacy assessment}

Using the posterior sample, for each patient of the test set, mean curve and $90 \%$-credibility intervals for predictions of body mass and caffeine concentration have been constructed (from Monte Carlo simulations) as a function of time (Subject 2 is the only plotted on Figure 4). For all the subjects of the test set, most of the caffeine concentration data fall in the credibility interval and body masses data are well estimated.

The empirical percentages of data points falling within different credibility intervals are close to the credibility percent coverage (last line of Table III), showing that the shapes of the statistical distributions chosen to model measurement errors are appropriate. The fact that observed percentages for caffeine concentration tend to be lower than the theoretical ones in the tails could 
be explained by the small number of caffeine concentration data points available in the test set ( $\mathrm{N}=21$ for 11 subjects).

\section{Comparison of Predictions from Previously Published Caffeine PK Models}

Three population PK models for caffeine have been previously proposed ${ }^{[1-3]}$. Caffeine concentration predictions for data belonging to the test set are made using these three models. Two of these models (Lee's and Falcao's) cannot predict body masses of neonates. Using set body masses (as observed at birth, for example) would be very penalizing for these models, so we choose to use the actually measured body masses, which is to their advantage. Monte Carlo simulations are run on the basis of the published parameter distributions to obtain credibility intervals on caffeine concentration predictions. For each model previously proposed average mean square errors (AMSE) and percentages of adequacy data points falling in different "theoretical" credibility intervals are given in Table II.

[Table II about here]

Our model better assesses uncertainty on caffeine concentration predictions: observed percentages of data points falling in the "theoretical" credibility intervals are better than those obtained with previous models. These models have higher bias or over-dispersion (higher AMSE) than our model. Relative residuals $\left(\frac{Y_{o b s}-\bar{Y}}{Y_{o b s}}, Y_{o b s}\right.$ observations and $\bar{Y}$ the estimate) of each model are plotted against postnatal age on Figure 5. It appears that our model gives a systematic prediction error at early time point (between the third and the eighth day of treatment). This point is discussed further below. However, most of the times, the proposed model gives better mean predictions than the other models for this data set.

[Figure 5 about here] 


\section{Recalibration}

Again, three chains of 60, 000 iterations are run. One out of 10 of the last 40, 000 simulations of the three chains are recorded, yielding 12, 000 parameter sets. The Gelman-Rubin convergence diagnostic is less than 1.06 for all parameters. Using this very final sample, summary statistics for marginal posterior distributions of the population parameters are given in Table III.

[Table III about here]

Compared to posteriors obtained during the first calibration step (involving 24 subjects), the estimates have not drastically changed. Estimated statistics (means and standard deviations) of population parameters have changed by less than $33 \%$. Parameters related to $\alpha_{1}$ and $C L_{0}$ are the most significantly updated. $\mu_{\alpha 1}$ is higher than previously; $\sigma_{\alpha 1}, \mu_{C L O}$ and $\sigma_{C L O}$ have also increased. The estimated uncertainty of these parameters is slightly higher than after the initial calibration step. This is due to fact that the estimates of the individual parameters $C L_{0}$ and $\alpha_{1}$ for the subjects of the test set are not fully centered in the previous population distribution.

\section{Sequential Updating of Predictions Using Sequential Incoming Data}

Obviously, more accurate predictions and credibility intervals would arise from taking into account the incoming data (body masses, blood samples, etc.) about subjects as they are clinically followed. This sequential update can easily be performed by correcting the previous posterior distributions using the new data. Since this update would not induce a drastic change, it is not necessary to run new MCMC chains. We demonstrate here the use of an efficient way to do it via particle methods ${ }^{[40]}$.

Let us illustrate the method through an example. Sequential updating is performed for patient 31 (of the test set). We examine how different kinds of data can improve caffeine concentration predictions. The update is first done using mass data, then using caffeine concentration data. The 
update could also be done with both kinds of data (mass and caffeine concentration data); the procedure would be same. Performing update with body mass data and caffeine concentration data separately allows to illustrate how informative caffeine concentration data are in comparison with body mass data.

Neonate 31 is born at $A_{0}=30$ weeks, with body mass $M_{0}=1.575 \mathrm{~kg}$. The daily body masses were measured during the 9 first days of treatment (Figure 6). The update is implemented first on the basis of recorded mass, using an original posterior sample of size of 12,000. An updated parameters sample is then obtained.

The updated posterior distributions for $\alpha_{1}, \alpha_{1}$ and $\tau$ are more accurate than the initial guess. Body masses dramatically increase knowledge about the time evolution pattern of mass for the patient. Figure 6 shows, for that subject, the predicted masses generated from the original posterior sampled parameters (panel A) and the predictions generated from updated parameters (panel B). Masses are clearly much better predicted in panel B. This updating procedure took about sixty seconds (on a pentium 4, microprocessor PC 2.8GHz running Windows XP), whereas running the three chains of the calibration procedures took about one day. The proposed updating procedure is about 1500 time faster than MCMC initial calibration.

[Figure 6 about here]

It is interesting to see how update can improve caffeine concentration predictions. Caffeine concentration in blood was measured for this subject on the third day of treatment $(\mathrm{c}=5.2, \mathrm{mg} / \mathrm{L})$. The predictions derived from the original posterior distribution are quite bad (Figure 7A) and the predictions obtained from updated sample using the only body mass data are not good either (Figure 7B). Whereas predictions for masses are much better after mass parameter updating (Figure 6), caffeine concentration predictions on Figure 7B seems unchanged (the distribution tails below $5 \%$ tile and above the $95 \%$ tile do change in fact). 
The same updating method is then performed using only the caffeine concentration observation (Figure 7C). The concentration data now falls within the $90 \%$-credibility interval and the mean estimate $(9.7 \mathrm{mg} / \mathrm{L})$ is also nearer the data after updating than before $(17.6 \mathrm{mg} / \mathrm{L})$. The concentration data is very informative relatively compared to the original posterior, especially on clearance, as expected. The gain on precision of predictions brought by the updating using caffeine concentration data can be measured by comparing the spread of the $90 \%$-credibility interval on caffeine concentration predictions before and after the update. The mean range between the lower and the upper bounds of the credibility interval (respectively corresponding to the 5 th and the 95th \%tiles) is equal to $26.2 \mathrm{mg} / \mathrm{L}$ before updating, $25.9 \mathrm{mg} / \mathrm{L}$ after updating using mass data and has been reduced to $13.2 \mathrm{mg} / \mathrm{L}$ after updating using caffeine concentration data.

[Figure 7 about here]

$C L_{0}$ marginal updated distribution for individual'31 has been transformed (in shape and mean) after updating (the mean of $C L_{0}$ has changed from 0.017 to 0.033 and the standard deviation has staid around 0.008 ). On the other hand, $\gamma$ distribution has not been much revised (the average of $\gamma$ has changed from $1.043 \times 10^{-5}$ to $1.087 \times 10^{-5}$ and the standard deviation from $0.889 \times 10^{-5}$ to 1.020 $\left.\times 10^{-5}\right)$.

An important concern is to decide when to update the distributions. It would not be optimal to do it every day, for example after each body weight measurement, because of the low sensitivity of caffeine elimination parameters to those measurements. A pragmatic general answer can be found in Chopin $2002^{[40]}$. In our case, a reasonable choice might be to perform the updating as soon as the observed body mass goes out of the predictions' (90\%) credibility interval. Moreover, since the caffeine concentration data are strongly informative, it seems that update should be done after each new caffeine concentration measurement. 
The updating procedure improves predictions for an individual as new data are collected, and thus, allows to guide dosing for this particular patient taking into account all its physiological characteristics (given by the covariates and the data). At the same time, this procedure also updates the joint population parameters distributions since MCMC calibration produces samples from the overall joint distribution of population and individual parameters. Figure 8 shows histograms of the marginal updated distributions for population mean and standard deviation of $C L_{0}$ and $\gamma$. As only one caffeine concentration data point on one subject has been used to update these population parameters, their distributions have not drastically changed. Nevertheless, $\mu_{C L O}$ and $\sigma_{C L O}$ distributions have been slightly translated to the right after updating, corresponding to greater values. This is coherent with the high value of $C L_{0}$ for that individual. On the other hand, $\sigma_{\gamma}$ distributions before and after updating shows that $\sigma_{\gamma}$ has been slightly reduced because the estimated $\gamma$ for the new subject is well centered with the original population distribution.

[Figure 8 about here]

\section{Discussion and Perspectives}

The present study aimes at providing the methodological basis for a quantitative decision support software for pediatricians who would like to anticipate neonates' caffeine concentration in blood, for a given treatment. Fitting a population model to individual data allowed us to make predictions for new subjects, given their covariates at birth, and a sample of random vectors of parameters from the posterior population distribution. These simulations give an "average prediction" for future caffeine concentrations during treatment, as well as credibility intervals for them. We present a fast and easy way to update those predictions on the basis of subject specific data on body mass and caffeine concentration in blood. 
Three caffeine distribution population models have already been proposed ${ }^{[1-3]}$. Thomson et al.'s model assumes that clearance depends on body mass at birth and does not account for mass changes over time. Similarly, caffeine distribution volume is also assumed to stay constant during treatment period. Making predictions over weeks, in such a context, can be misleading since the physiological variable of the premature neonates change significantly during the first trimester of life. Falcao et al. and Lee et al.'s models consider parameters (clearance and volume) as dynamic but requires infant body mass values at the time at which caffeine concentration is to be predicted. Lacking a body mass model, such models cannot be used for predictions over several days or weeks. However, even with a perfect mass model (i.e. using actually measured body masses), these two models do not give predictions as good as those of our new model (see Figure 5). Overall, the model we propose gives the best predictions for our neonate population. Two points need however to be stressed:

- Its mean predictions are still quite far from some data points (7 over 21 relative residuals errors are over 50\%) revealing that the model might still be improved. Moreover, relative residual errors show a trend with post-natal age. Predictions of all four models over estimates just after birth and underestimats after about 25 days of postnatal age (Figure 5). This implies the models are not able to capture all information contained in the data.

- Our model works quite well with local (Amiens) neonates. We do not know if it would be the best at describing Thomson's, Lee's or Falcao's data. We would recommend for a new calibration when going to new populations.

The population variability in caffeine clearance is relatively large and is the cause of the relatively large width of credibility intervals for the caffeine concentration predictions. This actually reflects the daily reality met by physicians. Part of this variability might be explained by introducing covariates which have not been taken into account here (as caffeine or tobacco 
maternal consummation during pregnancy, other administered treatment than caffeine, for example).

Another improvement could concern the model itself, and more precisely the absorption model. We made the assumption that oral absorption is very rapid. This is questionable. Not describing precisely the absorption process could generate errors in predictions at early time points. Given that misspecification the model could over-estimates the speed of caffeine bioavailability after the third day (beginning of per os administration). This over-estimation could lead to an underdosing over that period. A first-order oral absorption model might be better in this situation. However, after about the eighth day, caffeine absorption by oral route seems more or less quite well represented with a zero-order absorption rate (see Figure 5). We also made some choices which could be reconsidered, such as the linear form for the temporal evolution of physiological parameters, or the relative error to model the intra-individual variability in the body mass. Nonparametric modeling ${ }^{[49,50]}$ of population distribution could also be an interesting approach. Nonparametric models would relax hypotheses on parameter distributions. They could allow identification, for example, of bimodal population distributions, which could help in determining new explanatory covariates.

As different updating methods exist, it could be interesting to compare the different approaches of sequential updating (MAP Bayesian, Multiple Model, and Interacting Multiple Model). It would also be interesting to compare our method to other ones ${ }^{[23-25]}$ already proposed for dose individualization by applying them to caffeine dosing, for example.

A therapeutic dosing support tool should incorporate a sequential updating procedure such as the one detailed before. Admittedly, our procedure needs to be tuned (jump function, number of particle $)^{[40]}$ but it is fast, automatic and can be programmed easily in a medical support tool (currently under development). The only requirement for the user is to enter the newly collected 
data. The pediatrician does not need to know details of modeling or statistics. The therapeutic support tool would, then, give individualized predictions on the basis of information collected on the treated patient. The updating using caffeine concentration data can give better estimate (less biased) and as the same time, reduce the spread of credibility interval of caffeine concentration predictions (by around a factor 2, in our example). The proposed particle algorithm allows to quickly update individual parameters distribution. Moreover, population parameters distributions are updated simultaneously so that all available information from past or current patients will be available for any new subject entering the hospital.

To validate the benefit of the use of such a pharmacokinetic tool, a clinical trial could be based on a judgement criteria including observed effects after the comparison of two patient groups: one receiving the usual dosing and another one for which the dosing is assisted by the software tool. Finally, this technique was applied to caffeine as a demonstration study but could also be applied to other drugs with a narrower therapeutic window. Indeed, that would be all the more useful. Drugs for which the target serum concentration is close to the concentration limit leading to severe adverse effects, would certainly benefit to this method since it allows a quick and individualized estimation of the drug concentration using a limited number of measurements. 


\section{Reference List}

1. Thomson AH, Kerr S, Wright S. Population pharmacokinetics of caffeine in neonates and young infants. Ther Drug Monit 1996;18(3):245-53.

2. Lee TC, Charles B, Steer P, Flenady V, Shearman A. Population pharmacokinetics of intravenous caffeine in neonates with apnea of prematurity. Clin Pharmacol Ther 1997;61(6):628-40.

3. Falcao AC, Fernandez de Gatta MM, Delgado Iribarnegaray MF, Santos Buelga D, Garcia MJ, Dominguez-Gil A, et al. Population pharmacokinetics of caffeine in premature neonates. Eur J Clin Pharmacol 1997;52(3):211-7.

4. Daily WJ, Klaus M, Meyer HB. Apnea in premature infants: monitoring, incidence, heart rate changes, and an effect of environmental temperature. Pediatrics 1969;43(4):510-8.

5. Aranda JV, Turmen T, Davis J, Trippenbach T, Grondin D, Zinman R, et al. Effect of caffeine on control of breathing in infantile apnea. J Pediatr 1983;103(6):975-8.

6. Aranda JV, Gorman W, Bergsteinsson H, Gunn T. Efficacy of caffeine in treatment of apnea in the low-birth-weight infant. J Pediatr 1977;90(3):467-72.

7. Aranda JV, Grondin D, Sasyniuk BI. Pharmacologic considerations in the therapy of neonatal apnea. Pediatr Clin North Am 1981;28(1):113-33.

8. Zanardo V, Dani C, Trevisanuto D, Meneghetti S, Guglielmi A, Zacchello G, et al. Methylxanthines increase renal calcium excretion in preterm infants. Biol. Neonate 1995;68(3):169-174.

9. Gillot I, Gouyon JB, Guignard JP. Renal effects of caffeine in preterm infants. Biol. Neonate 1990;58:133-136.

10. D'Urzo AD, Jhirad R, Jenne H, Avendano MA, Rubinstein I, D'Costa M, et al. Effect of Caffeine on ventilatory responses to hypercapnia, hypoxia, and exercice in humans. J. Appl. Physiol. 1990;68(1):322-328.

11. Aranda JV, Clozel M. Pharmacologic effects of caffeine and theophylline in the premature infant. Dev Pharmacol Ther 1982;4 Suppl:165-72.

12. Karacan I, Thornby JI, Anch M, Booth GH, Williams RL, Salis PJ. Dose-related sleep disturbances induced by coffee and caffeine. Clin. Pharmacol. Ther. 1976;20(6):682-689.

13. Henderson-Smart DJ, Steer P. Methylxanthine treatment for apnea in preterm infants. Cochrane Database Syst Rev 2000(2):CD000140.

14. Bhatt-Mehta V, Schumacher RE. Treatment of apnea of prematurity. Paediatr Drugs 2003;5(3):195-210.

15. Box GEP, Tiao G. Bayesian Inference in Statistical Analysis. New York; 1973.

16. Gelman A, Carlin JB, Stern HS, Rubin DB. Bayesian Data Analysis. London: Chapman \& Hall; 1995.

17. Bernardo JM, Smith AFM. Bayesian Theory. New York: Wiley; 1994.

18. Amzal B, Bois FY, Parent E, Robert CP. Bayesian optimal design via interacting particle systems. Journal of the American Statistical Association 2006; 101(474):773-785.

19. Warszawski D, Gorodischer R. Tissue distribution of caffeine in premature infants and in newborn and adult dogs. Biol. Neonate 1981;1.

20. International Commission on Radiological Protection (ICRP). Basic Anatomical and Physiological data for Use in Radiological Protection: Reference Values. Stockholm, Sweden: Pergamon; 2002. 
21. Pons G, Carrier O, Richard M-O, Rey E, D'Athis P, Moran C, et al. Develppmental changes of caffeine elimination in infancy. Dev Pharmacol Ther 1988;11:258-264.

22. Carrier O, Pons G, Rey E, Richard MO, Moran C, Badoual J, et al. Maturation of caffeine metabolic pathways in infancy. Clin Pharmacol Ther 1988;44(2):145-51.

23. Bourgoin H, Paintaud G, Buchler M, Lebranchu Y, Autret-Leca E, Mentre F, et al. Bayesian estimation of cyclosporin exposure for routine therapeutic drug monitoring in kidney transplant patients

Nonparametric estimation of population characteristics of the kinetics of lithium from observational and experimental data: individualization of chronic dosing regimen using a new Bayesian approach. Br J Clin Pharmacol 2005;59(1):18-27.

24. Sandstrom M, Karlsson MO, Ljungman P, Hassan Z, Jonsson EN, Nilsson C, et al. Population pharmacokinetic analysis resulting in a tool for dose individualization of busulphan in bone marrow transplantation recipients. Bone Marrow Transplant 2001;28(7):657-64.

25. Taright N, Mentre F, Mallet A, Jouvent R. Nonparametric estimation of population characteristics of the kinetics of lithium from observational and experimental data: individualization of chronic dosing regimen using a new Bayesian approach. Ther Drug Monit 1994;16(3):258-69.

26. Arnaud M. Metabolism of caffeine and other components of coffee. In: Coffee and Health. New York: S Garattini; 1993. p. 43-93.

27. Aranda JV, Collinge JM, Zinman R, Watters G. Maturation of caffeine elimination in infancy. Arch Dis Child 1979;54(12):946-9.

28. Le Guennec JC, Billon B, Pare C. Maturational changes of caffeine concentrations and disposition in infancy during maintenance therapy for apnea of prematurity: influence of gestational age, hepatic disease, and breast-feeding. Pediatrics 1985;76(5):834-40.

29. Beal SL, Sheiner LB. Estimating population kinetics. Crit Rev Biomed Eng 1982;8(3):195-222.

30. Racine-Poon A, Smith AF. Population models. In: Berry DA, editor. Statistical Methodology in the Pharmaceutical Sciences. New York: Marcel Dekker, Inc.; 1990. p. 139-162.

31. Garthwaite PH, Kadane JB, O'Hagan A. Statistical methods for eliciting probability distributions. Journal of the American Statistical Association 2005;100(470):680-700.

32. Kadane JB, Wolfson LJ. Experiences in elicitation. Journal of the Royal Statistical Society Series D-the Statistician 1998;47(1):3-19.

33. Chen MH, Ibrahim JG, Shao QM, Weiss RE. Prior elicitation for model selection and estimation in generalized linear mixed models. Journal of Statistical Planning and Inference 2003;111(1-2):57-76.

34. Arnaud M, Weitzholtz H, Voegelin M, Bircher J, Presig R. Assessment of the cytochrome P-448 dependent liver enzyme system by caffeine breath test. In: sato R, editor. Microsomes drug oxydation and drug toxicity. New York: Wiley Interscience; 1982. p. 443-444.

35. Stones. Cross-validation choice and assessment of statistical predictions. Journal of the Royal Statstical Society B 1974;36:111-147.

36. Gelfand AE, Dey DK, Chang H. Model determination using predictive distributions with implementation via sampling-based methods. In: Bernardo JM, Berger JO, Dawid AP, Smith AFM, editors. Bayesian Statistics 4. Oxford: Oxford University Press; 1992. p. 147-167.

37. Robert C. Méthodes de Monte Carlo par Chaînes de Markov. Paris: Economica; 1996.

38. Gilks WR, Richardson S, Spiegelhalter DJ. Markov Chain Monte Carlo in Practice. London: Chapman \& Hall; 1996. 
39. Bois FY, Maszle D. MCSim: a simulation program. Journal of Statistical Software 1997;2(9):http://toxi.ineris.fr/activites/toxicologie_quantitative/mcsim/mcsim.php.

40. Chopin N. A sequential particle filter method for static models. Biometrika 2002;89:539552.

41. Tierney L, Mira A. Some adaptive Monte Carlo methods for Bayesian inference. Statistics in Medicine 1999;18: 2507-2515.

42. Doucet A, Godsill S, Andrieu C. On sequential Monte Carlo sampling methods for Bayesian filtering. Statistics and Computing 2000;10(3):197-208.

43. Doucet A, De Freias J, Gordon N. Sequential Monte Carlo Methods in Practice. New York: Springer-Verlag; 2001.

44. Ridgeway G, Madigan D. A sequential Monte Carlo method for Bayesian analysis of massive datasets. Data Mining and Knowledge Discovery 2003;7(3):301-319.

45. Bayard DS, Jelliffe RW. A Bayesian approach to tracking patients having changing pharmacokinetic parameters. Journal of Pharmacokinetics and Pharmacodynamics 2004;31(1):75-107.

46. Geweke J. Bayesian inference in econometric models using Monte Carlo integration. Econometrica 1989;24:1317-1399.

47. Rubin DB. Using the SIR algorithm to simulate posterior distributions. In: Bernardo JM, De Groot MH, Lindley DV, Smith AFM, editors. Bayesian Statistics 3. Oxford: Oxford University Press; 1988. p. 395-402.

48. Tierney L. Markov chains for exploring posterior distributions. Annals of Statistics 1994;22(4):1701-1762.

49. Jelliffe R, Schumitzky A, Van Guilder M. Population pharmacokinetics/pharmacodynamics modeling: parametric and nonparametric methods. Ther Drug Monit 2000;22(3):354-65.

50. Wakefield J, Walker S. Bayesian nonparametric population models: formulation and comparison with likelihood approaches. J Pharmacokinet Biopharm 1997;25(2):235-53. 


\section{Tables}

Table I: Parameter distributions of our PK model. Summary of the parameter distributions at individual level of the PK model and population prior distributions used in the calibration process.

\begin{tabular}{|c|c|c|}
\hline Parameter (unit) & Distribution & Truncation bounds \\
\hline \multicolumn{3}{|l|}{ Individual level } \\
\hline$\alpha_{1}(\mathrm{~kg} / \mathrm{h})$ & Normal $\left(\mu_{\alpha 1}, \sigma_{\alpha 1}\right)$ & {$\left[-5 \times 10^{-3} ; 0\right]$} \\
\hline$\alpha_{2}(\mathrm{~kg} / \mathrm{h})$ & Normal $\left(\mu_{\alpha 2}, \sigma_{\alpha 2}\right)$ & {$\left[0 ; 3 \times 10^{-3}\right]$} \\
\hline$\tau(\mathrm{h})$ & $\operatorname{LogNormal}^{1}\left(\mu_{\tau}, \sigma_{\tau}\right)$ & {$[72 ; 480]$} \\
\hline$f_{25}(\mathrm{~L} / \mathrm{kg})$ & $\operatorname{LogNormal}^{1}\left(\mu_{f 25}, \sigma_{f 25}\right)$ & {$[0.8 ; 0.9]$} \\
\hline$f_{\tau}(\mathrm{L} / \mathrm{kg})$ & $\operatorname{LogNormal}^{1}\left(\mu_{f \tau} ; \sigma_{f \tau}\right)$ & {$[0.7 ; 0.8]$} \\
\hline$C L_{0}(\mathrm{~L} / \mathrm{h})$ & $\operatorname{LogNormal}^{1}\left(\mu_{C L O}, \sigma_{C L O}\right)$ & {$[0 ; 0.06]$} \\
\hline$\gamma\left(\mathrm{L} / \mathrm{h}^{2}\right)$ & $\operatorname{LogNormal}^{1}\left(\mu_{\gamma}, \sigma_{\gamma}\right)$ & {$\left[5 \times 10^{-7} ; 10^{-4}\right]$} \\
\hline$c v_{M}($ unitless $)$ & Normal $\left(\mu_{c v M}, \sigma_{c v M}\right)$ & {$[0.010 ; 0.100]$} \\
\hline \multicolumn{3}{|l|}{ Population level } \\
\hline$\mu_{\alpha 1}(\mathrm{~kg} / \mathrm{h})$ & Normal $\left(-10^{-3}, 0.6 \times 10^{-3}\right)$ & {$\left[-5 \times 10^{-3} ; 0\right]$} \\
\hline$\mu_{\alpha 2}(\mathrm{~kg} / \mathrm{h})$ & Normal $\left(10^{-3}, 0.5 \times 10^{-3}\right)$ & {$\left[0 ; 3 \times 10^{-3}\right]$} \\
\hline$\mu_{\tau}(\mathrm{h})$ & Uniform & {$[72 ; 480]$} \\
\hline$\mu_{f 25}(\mathrm{~L} / \mathrm{kg})$ & Normal $(0.85,0.05)$ & {$[0.8 ; 0.9]$} \\
\hline$\mu_{f \tau}(\mathrm{L} / \mathrm{kg})$ & Normal $(0.75,0.05)$ & {$[0.7 ; 0.8]$} \\
\hline$\mu_{C L O}(\mathrm{~L} / \mathrm{h})$ & Normal $(0.02,0.01)$ & {$[0 ; 0.04]$} \\
\hline$\mu_{\gamma}\left(\mathrm{L} / \mathrm{h}^{2}\right)$ & Normal $\left(5 \times 10^{-6}, 5 \times 10^{-6}\right)$ & {$\left[5 \times 10^{-7} ; 10^{-4}\right]$} \\
\hline$\sigma_{\alpha 1}$ & $\operatorname{LogNormal}^{1}\left(0.4 \times 10^{-3}, 2.96\right)$ & - \\
\hline$\sigma_{\alpha 2}$ & $\operatorname{LogNormal}^{1}\left(0.4 \times 10^{-3}, 2.96\right)$ & - \\
\hline$\sigma_{\tau}$ & $\operatorname{LogNormal}^{1}(1.32,2.30)$ & {$[1.01 ; 2]$} \\
\hline$\sigma_{f 25}$ & LogNormal $^{1}(1.183,1.22)$ & {$[1.01 ; 2]$} \\
\hline$\sigma_{f \tau}$ & LogNormal $^{1}(1.183,1.22)$ & {$[1.01 ; 2]$} \\
\hline$\sigma_{C L O}$ & LogUniform & {$[1.1 ; 3]$} \\
\hline$\sigma_{\gamma}$ & LogUniform & {$[1.1 ; 3]$} \\
\hline$\mu_{c v M}$ (unitless) & Uniform & {$[0.010 ; 0.100]$} \\
\hline$\sigma_{c v M}$ & Normal $(0.030,0.020)$ & {$[0.005 ; 0.100]$} \\
\hline$\sigma_{C}$ & Normal $(1.105,0.1)$ & {$[1.01 ; 2]$} \\
\hline
\end{tabular}

${ }^{1}$ For LogNormal distributions, the parameters in parenthesis correspond to the geometric mean (exponential of the mean in log-space) and geometric standard deviation (exponential of the standard deviation in log-space). 
Table II: Indicators to compare the existing PK models. Average mean square error (AMSE) and percentages of adequacy data points falling within the "theoretical" credibility intervals given by various models. These data points concern caffeine concentration of the subjects belonging to the test set. 21 data points are available for caffeine concentration in blood.

\begin{tabular}{l|cccccc|c}
\hline \multirow{2}{*}{\multicolumn{1}{c|}{ Model }} & \multicolumn{6}{|c|}{ Nominal credibility interval coverage (\%) } & \multirow{2}{*}{ AMSE } \\
\cline { 2 - 7 } & 40 & 50 & 80 & 85 & 90 & 95 & \\
\hline Thomson's et $\boldsymbol{a l}$. & 4.8 & 4.8 & 33.3 & 33.3 & 38.1 & 38.1 & 1.358 \\
Lee's $\boldsymbol{e t}$ al. & 9.5 & 9.5 & 14.3 & 28.6 & 42.9 & 47.6 & 2.207 \\
Falcao's $\boldsymbol{e t}$ al. & 9.5 & 14.3 & 42.9 & 42.9 & 47.6 & 57.1 & 2.036 \\
New proposed model & 48.2 & 52.4 & 61.9 & 71.4 & 81.0 & 81.0 & 0.411 \\
\hline
\end{tabular}


Table III: Summary statistics for the ultimate $(\mathrm{N}=35$ subjects) marginal posterior distributions of the population parameters.

\begin{tabular}{|c|c|c|c|c|}
\hline Population parameters & mean & SD & $2.5 \%$ tile & 97.5\% tile \\
\hline$\overline{\mu_{\alpha 1}}$ & 0.00101 & 0.000174 & 0.00129 & 0.000592 \\
\hline$\mu_{\alpha 2}$ & 0.000948 & $4.21 \times 10^{-5}$ & 0.000864 & 0.00103 \\
\hline$\mu_{\tau}$ & 130 & 15.9 & 96.3 & 159 \\
\hline$\mu_{f 25}$ & 0.85 & 0.0215 & 0.808 & 0.892 \\
\hline$\mu_{f \tau}$ & 0.752 & 0.0213 & 0.708 & 0.793 \\
\hline$\mu_{C L O}$ & 0.0154 & 0.00183 & 0.0121 & 0.0192 \\
\hline$\mu_{\gamma}$ & $8.45 \times 10^{-6}$ & $2.43 \times 10^{-6}$ & $4.02 \times 10^{-6}$ & $1.35 \times 10^{-5}$ \\
\hline$\sigma_{\alpha 1}$ & 0.000676 & 0.000146 & 0.000464 & 0.00103 \\
\hline$\sigma_{\alpha 2}$ & 0.000237 & $3.19 \times 10^{-5}$ & 0.000185 & 0.000309 \\
\hline$\sigma_{\tau}$ & 1.6 & 0.145 & 1.38 & 1.93 \\
\hline$\sigma_{f 25}$ & 1.21 & 0.2 & 1.0 & 1.69 \\
\hline$\sigma_{f \tau}$ & 1.21 & 0.199 & 1.01 & 1.7 \\
\hline$\sigma_{C L O}$ & 1.54 & 0.198 & 1.2 & 2 \\
\hline$\sigma_{\gamma}$ & 1.91 & 0.456 & 1.17 & 2.86 \\
\hline$\mu_{c v M}$ & 0.0179 & 0.00462 & 0.0105 & 0.0269 \\
\hline$\sigma_{c v M}$ & 0.018 & 0.00332 & 0.012 & 0.025 \\
\hline$\sigma_{C}$ & 1.36 & 0.0483 & 1.28 & 1.47 \\
\hline
\end{tabular}




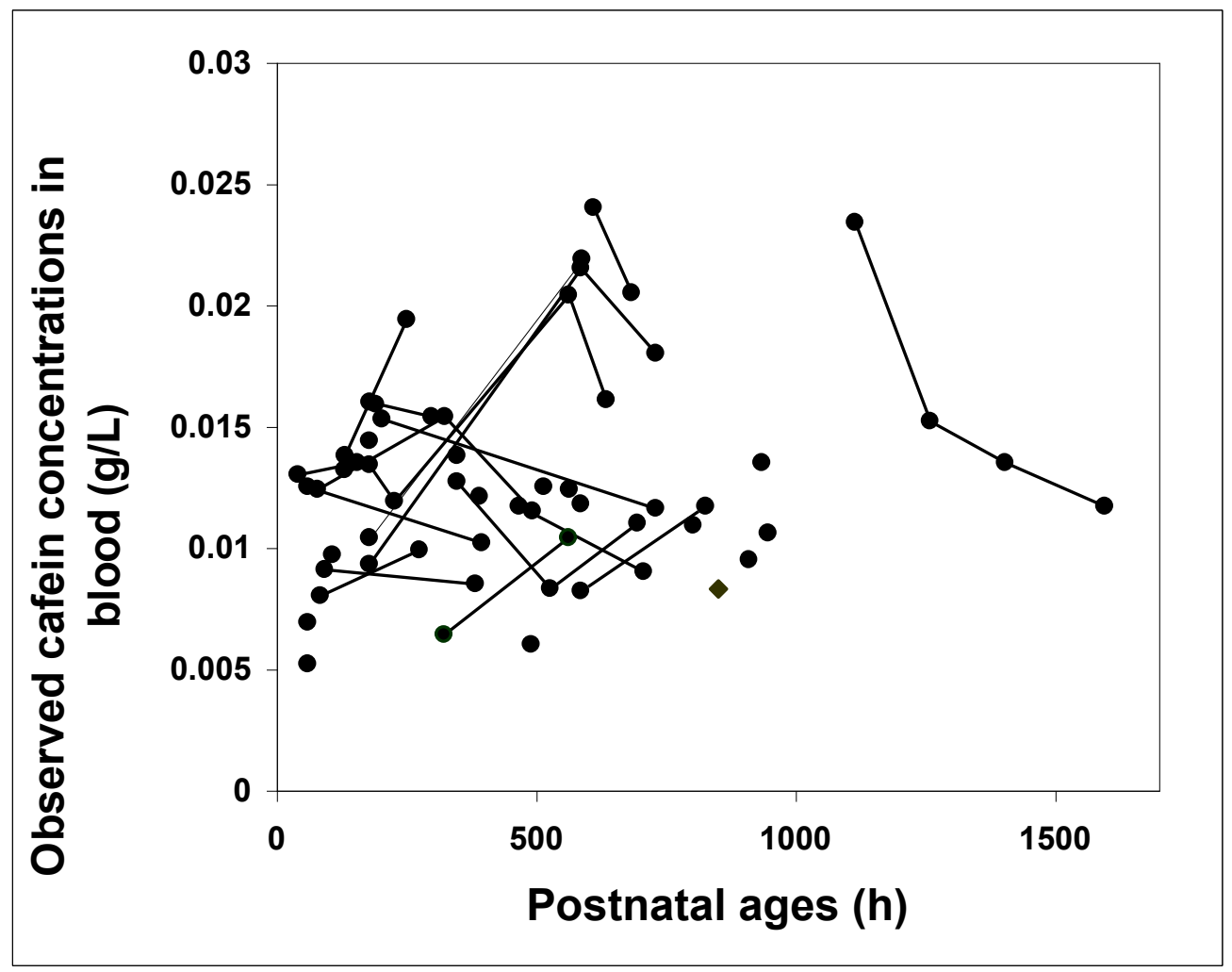

Figure 1: Observed caffeine concentrations in blood as a function of postnatal age. Joined points indicate observations belonging to a same individual. 


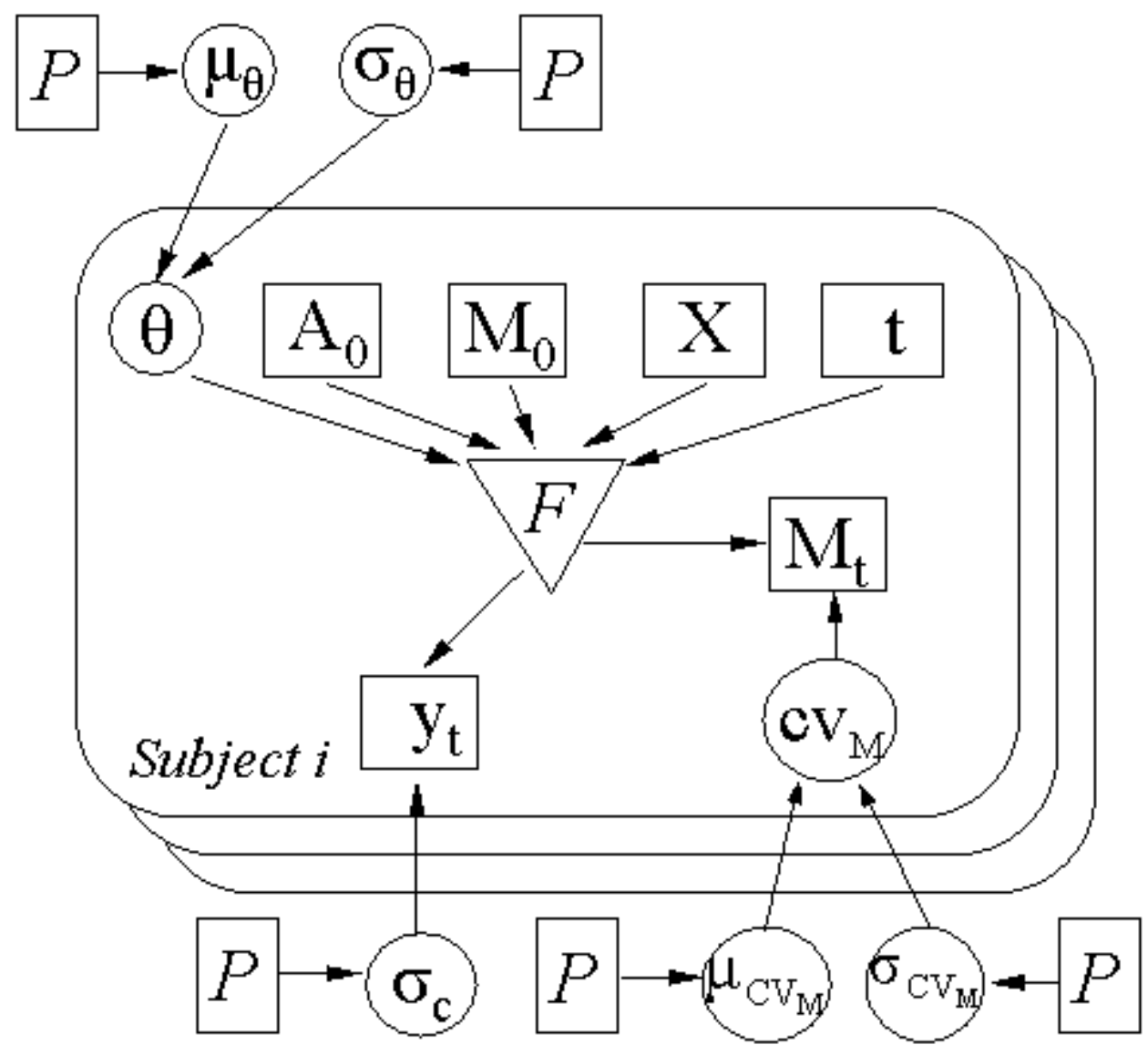

Figure 2: Directed acyclic graph of the hierarchical statistical model. Symbols are: $P$, prior distributions; $\mu_{\theta}$, population mean parameters; $\sigma_{\theta}$, variance of population parameter; $t$, observation times; $\theta$, unknown parameters; $A_{0}$ and $M_{0}$ are respectively gestational age and body mass at birth (both supposed exactly measured); $X$, the administered treatment; $M_{t}$, the measured body mass at time $t ; c v_{M}$, the variation coefficient of masses and $Y_{t}$, the measured caffeine concentration in blood. $\mu_{c v M}$, and $\sigma_{c v M}$ are respectively for mean and variance of $c v_{M}$ parameters and $\sigma_{c}$ for the variance of the experimental measurements. $F$ is the dynamic bio-distribution model. Square nodes are for variables of known (or supposed known) values; circular nodes for unknown variables and the triangle represents a deterministic link. 

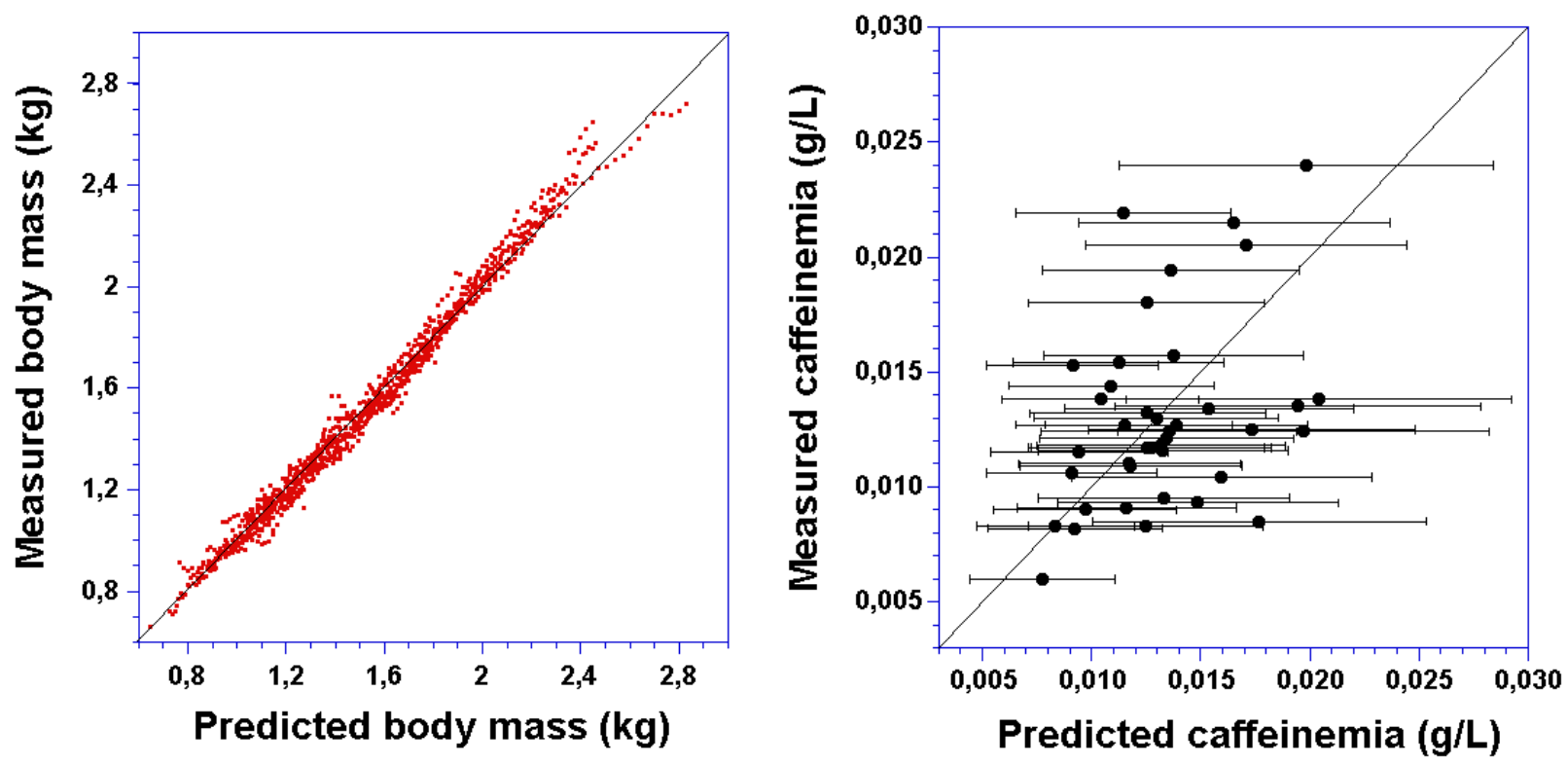

Figure 3: Data versus model predictions. The left and the right graphs respectively concern body masses and caffeine concentrations in blood for individuals of the training set. On the right side graph, each line segment describes the 90\%-credibility interval associated to the corresponding prediction. The 90\%-crediblity intervals have been plotted using $\sigma_{c}$ value of the maximum posterior vector. 

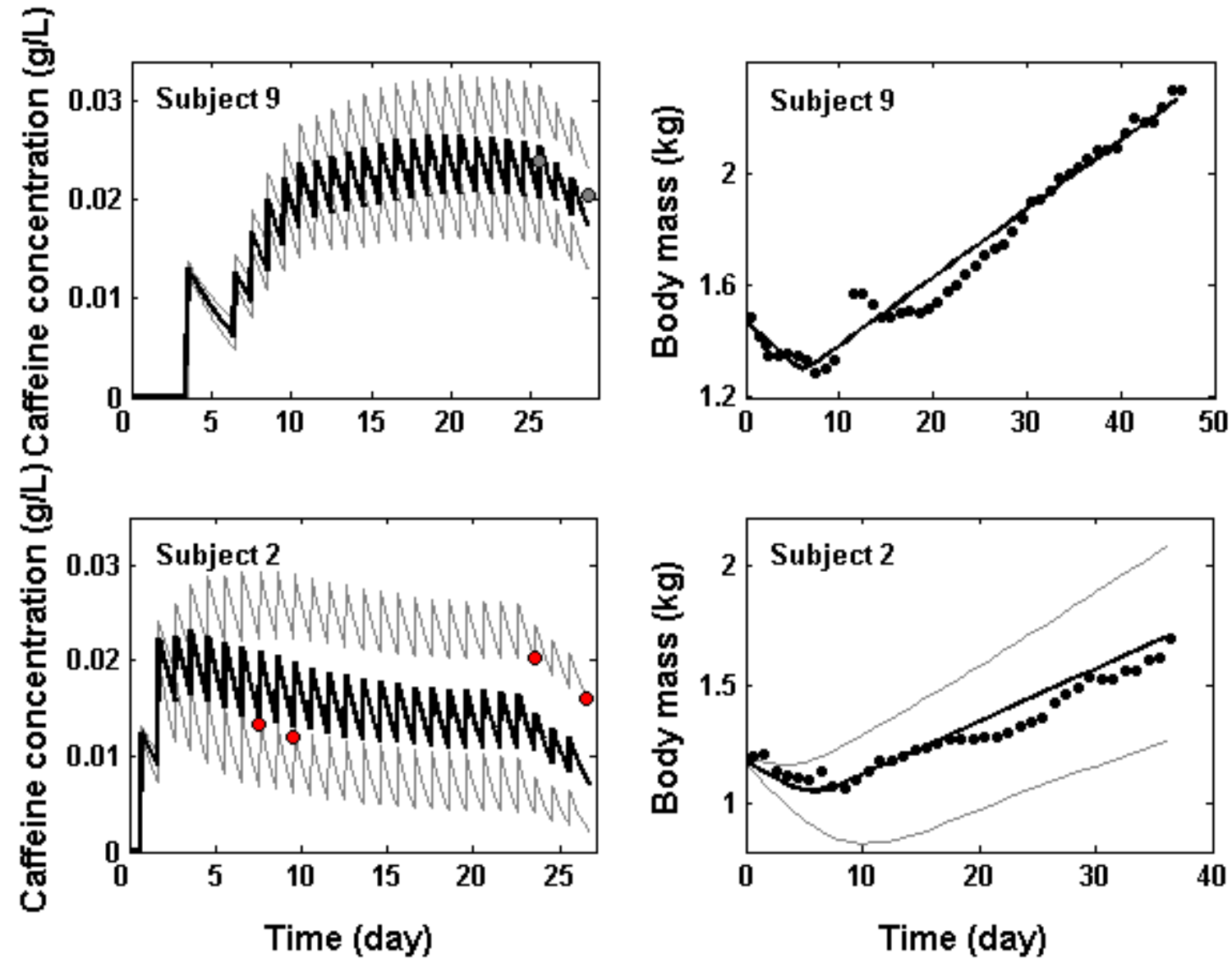

Figure 4: Caffeine concentration in blood (left) and body masses (right) as a function of time. Predictions have been calculated for one subject of the training set (Subject 9) and one subject of the test set (Subject 2). Means of the predictions are in bold lines, and the corresponding 90\%credibility intervals are plotted with thin lines. Data are represented by dots. Note that $90 \%$ credibility interval of the predicted masses is not represented for the subject of the calibration set (subject 9) as it was very thin. 


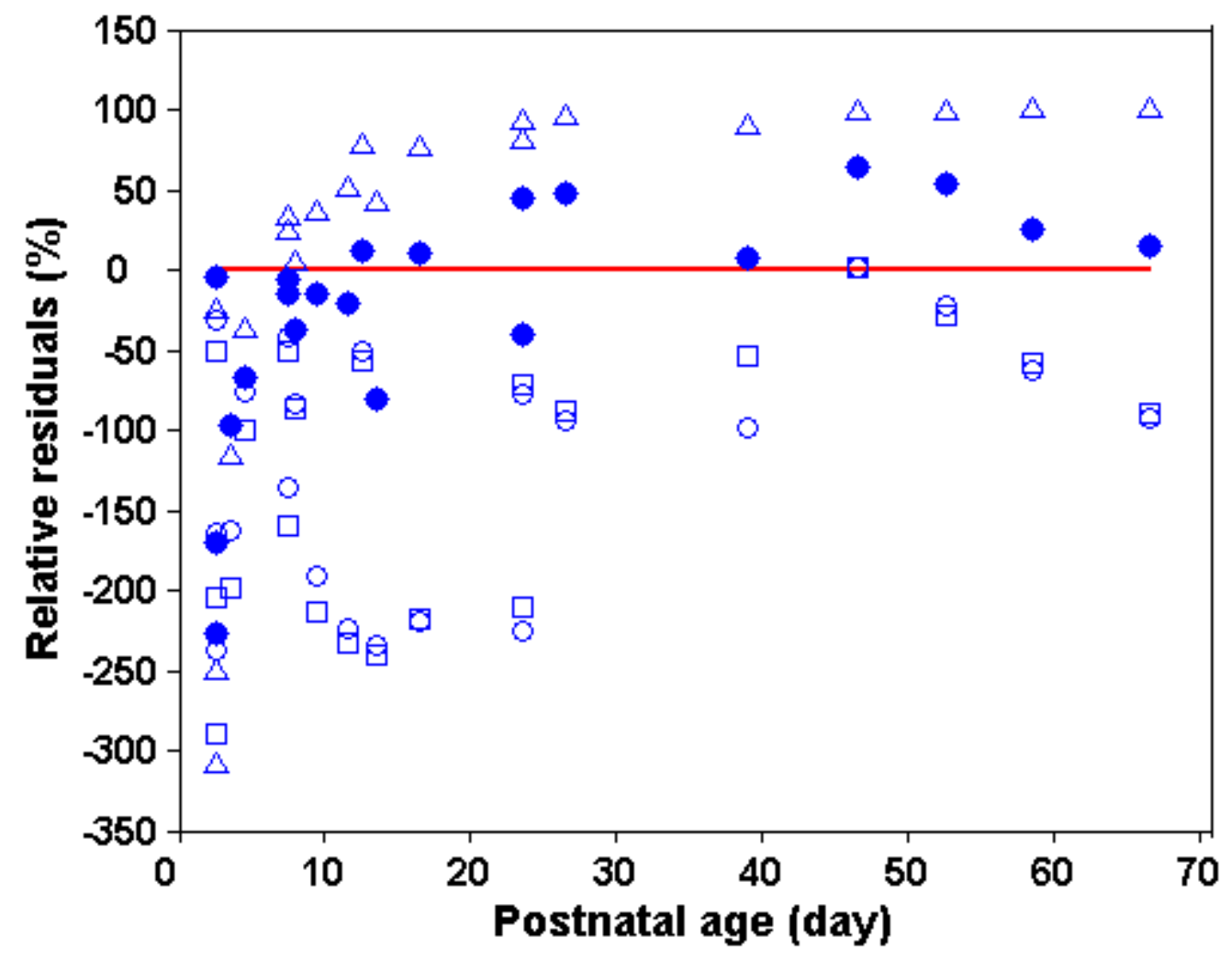

Figure 5: Relative residuals for caffeine concentration as a function of postnatal age. Four different models are represented: Thomson et. al. ${ }^{[1]}$ (triangles), Lee et. al. ${ }^{[2]}$ (squares), Falcao et. al. ${ }^{[3]}$ (empty circles) and the one we propose (plain circles). Relative residuals correspond to the relative differences between training set data points and mean caffeine concentration predictions obtained by Monte Carlo simulations. 


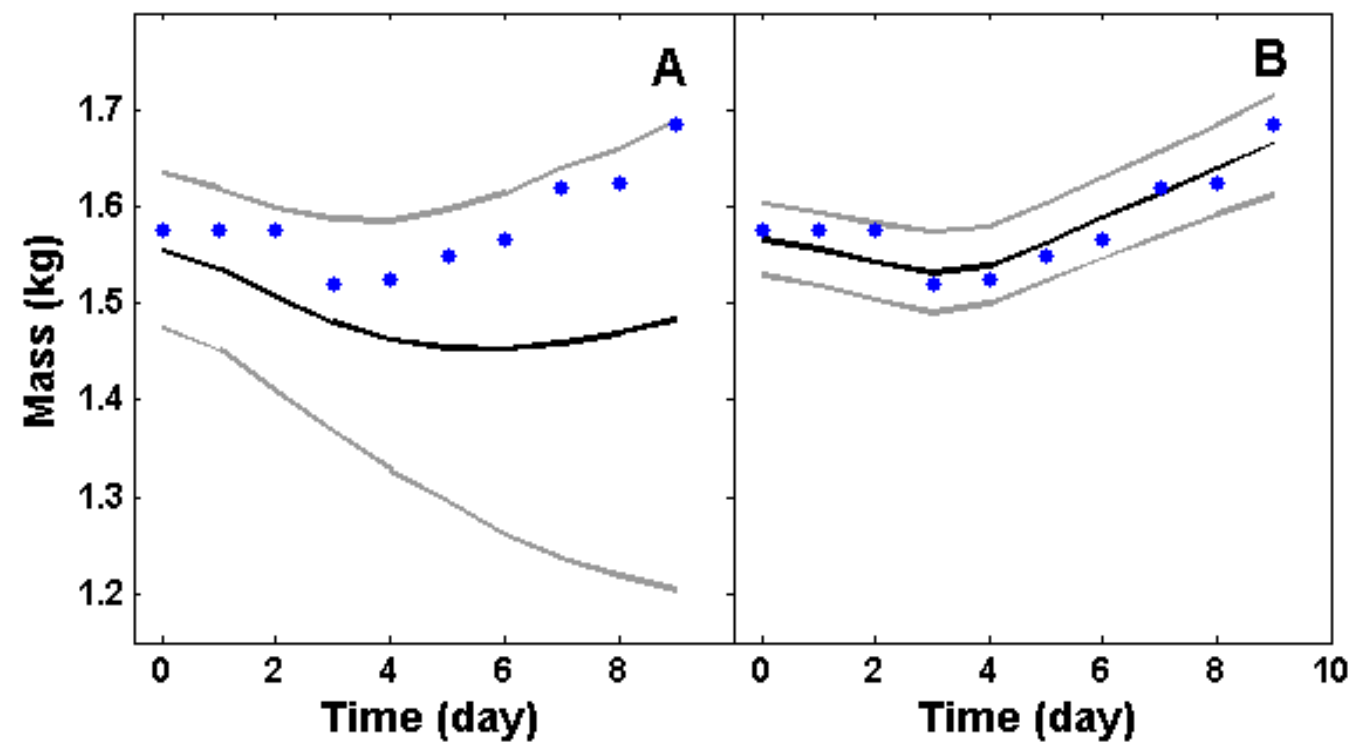

Figure 6: Body mass predictions as a function of the time for patient 31 . The $5 \%$, and $95 \%$ quantiles of predictions are plotted using gray lines, the mean of predictions using dark bold lines. Data are represented by the dots. Panel A shows the predicted masses calculated from the original posterior-sampled parameters, using the covariates of the patient at birth, only. Predictions of panel B use, in addition, body mass data via the updated posterior parameters obtained by a particle algorithm. 


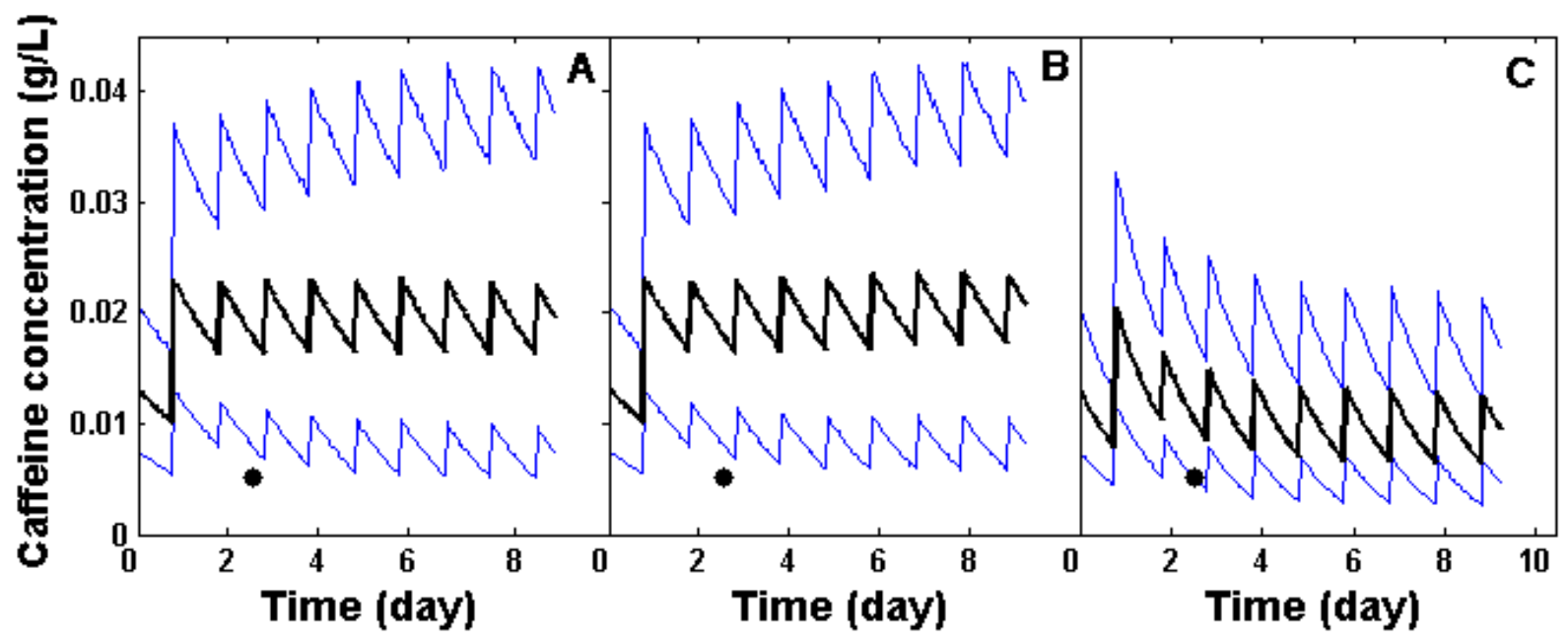

Figure 7: Caffeine concentration predictions as a function of time for patient 31 . The $5 \%$ and 95 $\%$ quantiles of predictions are plotted using thin lines, the mean of predictions using bold lines. The data point is represented by dot. Panel A shows the predicted caffeine concentration in blood calculated from original posterior sampled parameters and using only the covariates of the patient. Predictions of panel B and C are performed using the posterior parameters updated via a particle algorithm. In panel $\mathrm{B}$, only data masses were used for parameter updating while only measured caffeine concentration was used to update predictions in panel C. 

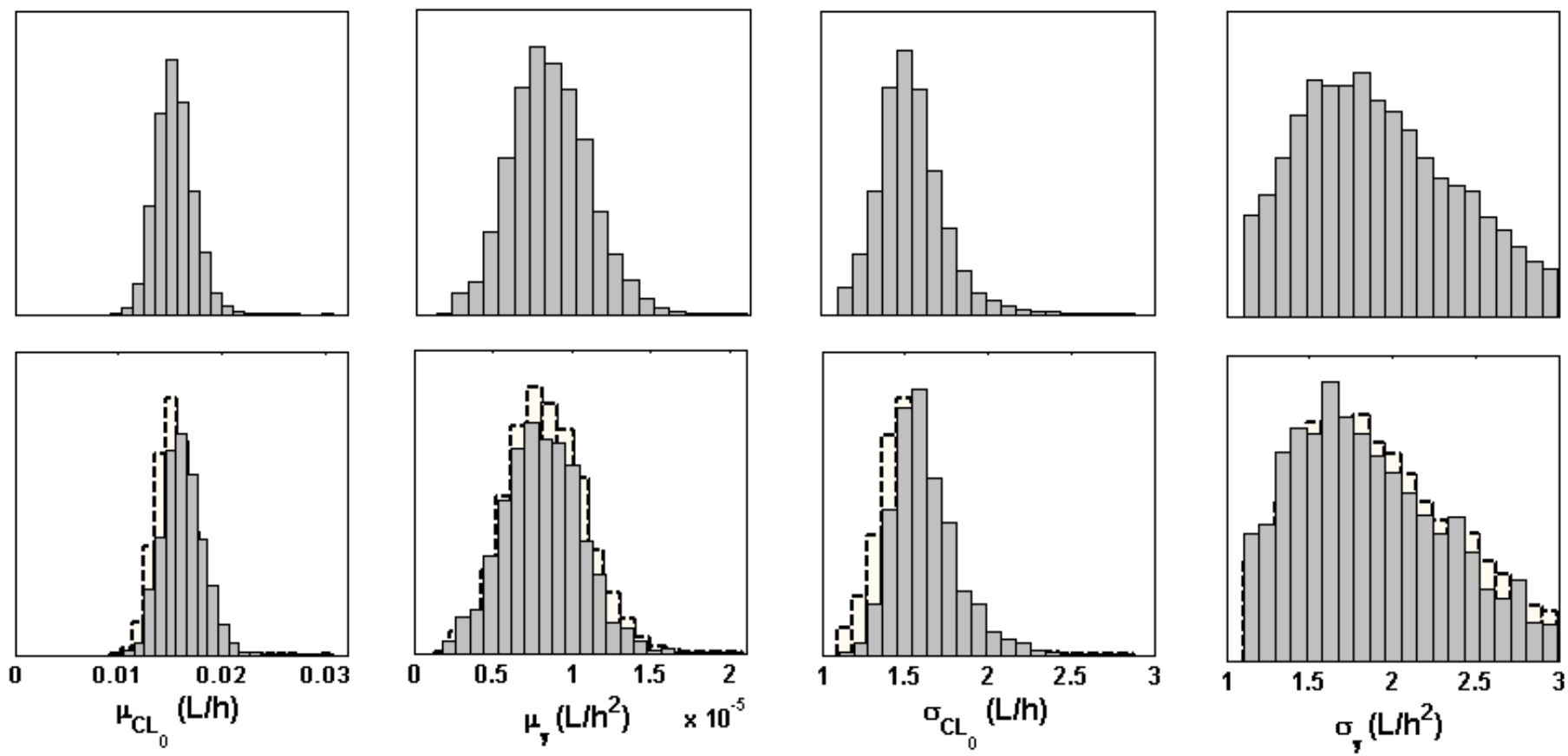

Figure 8: Histograms of the marginal posterior distributions of the population elimination model parameters. Top row graphs give the marginal distributions of the original posterior. Bottom row darker histograms give the marginal updated distributions, using one caffeine concentration data point for subject 31. Bottom row unfilled histograms recall the original posterior. 